\title{
Lack of exponential decay for a laminated beam with structural damping and second sound
}

\author{
Wenjun Liu, Xiangyu Kong and Gang Li \\ College of Mathematics and Statistics, Nanjing University of Information Science and Technology, \\ Nanjing 210044, China. E-mail: wjliu@nuist.edu.cn.
}

In previous work (Z. Angew. Math. Phys. 68(2), 2017), Apalara considered a onedimensional thermoelastic laminated beam under Cattaneo's law of heat conduction and proved the exponential and polynomial decay results depend on the stability number $\chi_{\tau}$. In this paper, we continue to study the same system and show that the solution of the concerned system lacks of exponential decay result in the case $\chi_{\tau} \neq 0$ which solves the open problem proposed by Apalara (Z. Angew. Math. Phys. 68(2), 2017).

Keywords: laminated beam, exponential stability, Cattaneo's law, semigroup theory

AMS Subject Classification (2000): 34B05, 35L05, 93C20, 93D20.

\section{Introduction}

In previous paper [1], Apalara considered the following laminated beam with structural damping and second sound:

$$
\begin{cases}\rho w_{t t}+G\left(\psi-w_{x}\right)_{x}=0, & (x, t) \in(0,1) \times(0,+\infty), \\ I_{\rho}\left(3 s_{t t}-\psi_{t t}\right)-D\left(3 s_{x x}-\psi_{x x}\right)-G\left(\psi-w_{x}\right)+\delta \theta_{x}=0, & (x, t) \in(0,1) \times(0,+\infty), \\ 3 I_{\rho} s_{t t}-3 D s_{x x}+3 G\left(\psi-w_{x}\right)+4 \gamma s+4 \beta s_{t}=0, & (x, t) \in(0,1) \times(0,+\infty), \\ \rho_{3} \theta_{t}+q_{x}+\delta\left(3 s_{t}-\psi_{t}\right)_{x}=0, & (x, t) \in(0,1) \times(0,+\infty), \\ \tau q_{t}+\alpha q+\theta_{x}=0, & (x, t) \in(0,1) \times(0,+\infty), \\ w(x, 0)=w_{0}(x), \psi(x, 0)=\psi_{0}(x), s(x, 0)=s_{0}(x), w_{t}(x, 0)=w_{1}(x), & x \in(0,1), \\ \psi_{t}(x, 0)=\psi_{1}(x), s_{t}(x, 0)=s_{1}(x), \theta(x, 0)=\theta_{0}(x), q(x, 0)=q_{0}(x), & x \in(0,1), \\ w_{x}(0, t)=\psi(0, t)=s(0, t)=q(0, t)=0, & t \in[0,+\infty), \\ w(1, t)=\psi_{x}(1, t)=s_{x}(1, t)=\theta(1, t)=0, & t \in[0,+\infty),\end{cases}
$$

where $\rho, G, I_{\rho}, \rho_{3}, D, \gamma, \beta, \delta, \alpha, \tau$ are positive constants. Here $w(x, t)$ denotes the transverse displacement of the beam which departs from its equilibrium position, $\psi(x, t)$ represents the rotation angle, $s(x, t)$ is proportional to the amount of slip along the interface at time $t$ and longitudinal spatial variable $x, 3 s-\psi$ denotes the effective rotation angle and the third equation of (1.1) describes the dynamics of the slip, $\theta(x, t)$ is the difference temperature, $q(x, t)$ is the heat flux. The coefficients $\rho, G, I_{\rho}, D, \gamma, \beta>0$ denote the density of the beams, the shear stiffness, the mass moment of inertia, the flexural rigidity, the adhesive stiffness of the beams, and the adhesive damping parameter, respectively. Moreover, the constants $\rho_{3}, \alpha, \delta>0$ represent the physical parameters from thermoelasticity theory, and $\tau$ denotes the relaxation time describing the time lag in the response of the heat flux to a gradient in the temperature. In that paper, the author 
established the exponential decay and polynomial decay depend on the stability number

$$
\chi_{\tau}=\left(1-\frac{\rho_{3} G \tau}{\rho}\right)\left(\frac{D}{I_{\rho}}-\frac{G}{\rho}\right)-\frac{\tau G \delta^{2}}{\rho I_{\rho}} .
$$

Beyond that, the author prove the well-posedness of the system. As for the previous results and developments of laminated beam, the authors have stated and summarized in great detail in [1], thus we just omit it here. The readers, for a better understanding of present work, are strongly recommended to see [1] and the references therein.

For the lack of exponential decay, there are some results in recent time. Apalara et al. [2] studied a one-dimensional thermoelastic Timoshenko system of the form

$$
\left\{\begin{array}{lr}
\rho_{1} \varphi_{t t}-k\left(\varphi_{x}+\psi\right)_{x}+\delta \theta_{x}=0, & (x, t) \in(0, L) \times(0,+\infty), \\
\rho_{2} \psi_{t t}-b \psi_{x x}+k\left(\varphi_{x}+\psi\right)=0, & (x, t) \in(0, L) \times(0,+\infty), \\
\rho_{3} \theta_{t}+q_{x}+\delta \varphi_{t x}=0, & (x, t) \in(0, L) \times(0,+\infty), \\
\tau q_{t}+q+\gamma \theta_{x}=0, & (x, t) \in(0, L) \times(0,+\infty),
\end{array}\right.
$$

where the heat conduction is given by Cattaneo's law and the coupling is via the displacement equation. The authors proved the exponential decay (polynomial decay) result when the stability number $\chi_{\tau}=0\left(\chi_{\tau} \neq 0\right)$. Moreover, when the stability number $\chi_{\tau} \neq 0$, they showed that the system lacks exponential stability if $\chi_{\tau}=0$ holds. In [8], Santos et al. studied a Bresse-Fourier system

$$
\left\{\begin{array}{lr}
\rho_{1} \varphi_{t t}-k\left(\varphi_{x}+\psi+l w\right)_{x}-k_{0} l\left(w_{x}-l \varphi\right)+k \theta_{x}=0, & (x, t) \in(0, L) \times(0,+\infty), \\
\rho_{2} \psi_{t t}-b \psi_{x x}+k\left(\varphi_{x}+\psi+l w\right)-k \theta=0, & (x, t) \in(0, L) \times(0,+\infty), \\
\rho_{1} w_{t t}-k_{0}\left(w_{x}-l \varphi\right)_{x}+k l\left(\varphi_{x}+\psi+l w\right)-k l \theta=0, & (x, t) \in(0, L) \times(0,+\infty), \\
\rho_{3} \theta_{t}-\alpha \theta_{x x}+k\left(\varphi_{x}+\psi+l w\right)_{t}=0, & (x, t) \in(0, L) \times(0,+\infty),
\end{array}\right.
$$

The authors proved the system is not exponentially stable when $\frac{k}{\rho_{1}} \neq \frac{b}{\rho_{2}}$ or $k-k_{0} \neq 0$. In addition, they established the exponential decay and polynomial decay deponed on the relation between the wave speed propagation. For more papers related to the lack of exponential stability, we refer the reader to $[3,4,5,7]$ and the references therein.

In [1], Apalara brought up an interesting open problem to study the lack of exponential stability of problem (1.1), that is, the system is not exponentially stable unless $\chi_{\tau}=0$. The aim of this paper is to give a positive answer to the open problem proposed by Apalara [1], that is we will prove the system is not exponentially stable unless stability number

$$
\chi_{\tau}=\left(1-\frac{\rho_{3} G \tau}{\rho}\right)\left(\frac{D}{I_{\rho}}-\frac{G}{\rho}\right)-\frac{\tau G \delta^{2}}{\rho I_{\rho}}=0 .
$$

More precisely, we will recall a standard and widely used technique for the investigation of the decay properties of an abstract contraction semigroup $S(t)=e^{\mathcal{A} t}$ acting on a Hilbert space $\mathcal{H}$.

The remaining part of this paper is organized as follows. In section 2, we present some hypotheses needed for our work and state the main result. In section 3 , we show that the system is not exponential stable if $\chi_{\tau} \neq 0$. We use $c$ throughout this paper to denote a generic positive constant. 


\section{Preliminaries and main results}

In this section, we begin with some materials and known result for problem (1.1). First, let $\xi=3 s-\psi$, then (1.1) can be rewritten as follows:

$$
\begin{cases}\rho w_{t t}+G\left(3 s-\xi-w_{x}\right)_{x}=0, & (x, t) \in(0,1) \times(0,+\infty), \\ I_{\rho} \xi_{t t}-D \xi_{x x}-G\left(3 s-\xi-w_{x}\right)+\delta \theta_{x}=0, & (x, t) \in(0,1) \times(0,+\infty), \\ 3 I_{\rho} s_{t t}-3 D s_{x x}+3 G\left(3 s-\xi-w_{x}\right)+4 \gamma s+4 \beta s_{t}=0, & (x, t) \in(0,1) \times(0,+\infty), \\ \rho_{3} \theta_{t}+q_{x}+\delta \xi_{x t}=0, & (x, t) \in(0,1) \times(0,+\infty), \\ \tau q_{t}+\alpha q+\theta_{x}=0, & (x, t) \in(0,1) \times(0,+\infty), \\ w(x, 0)=w_{0}(x), \xi(x, 0)=\xi_{0}(x), s(x, 0)=s_{0}(x), w_{t}(x, 0)=w_{1}(x), & x \in[0,1], \\ \xi_{t}(x, 0)=\xi_{1}(x), s_{t}(x, 0)=s_{1}(x), \theta(x, 0)=\theta_{0}(x), q(x, 0)=q_{0}(x), & x \in[0,1], \\ w_{x}(0, t)=\xi(0, t)=s(0, t)=q(0, t)=0, & t \in[0,+\infty), \\ w(1, t)=\xi_{x}(1, t)=s_{x}(1, t)=\theta(1, t)=0, & t \in[0,+\infty) .\end{cases}
$$

Next, let

$$
\Phi=(w, u, \xi, v, s, z, \theta, q)^{T}
$$

and

$$
\Phi_{0}(x)=\left(w_{0}, w_{1}, \xi_{0}, \xi_{1}, s_{0}, s_{1}, \theta_{0}, q_{0}\right)^{T},
$$

where $u=w_{t}, v=\xi_{t}$ and $z=s_{t}$. Hence, problem (2.1) is equivalent to the following abstract Cauchy problem:

$$
\left\{\begin{array}{l}
\partial_{t} \Phi(x, t)=\mathcal{A} \Phi(x, t), \\
\Phi(x, 0)=\Phi_{0}(x),
\end{array}\right.
$$

where $\mathcal{A}$ is a operator defined by

$$
\mathcal{A} U=\left(\begin{array}{c}
u \\
-\frac{G}{\rho}\left(3 s-\xi-w_{x}\right)_{x} \\
v \\
\frac{G}{I_{\rho}}\left(3 s-\xi-w_{x}\right)+\frac{D}{I_{\rho}} \xi_{x x}-\frac{\delta}{I_{\rho}} \theta_{x} \\
z \\
-\frac{G}{I_{\rho}}\left(3 s-\xi-w_{x}\right)-\frac{4 \gamma}{3 I_{\rho}} s-\frac{4 \beta}{3 I_{\rho}} z+\frac{D}{I_{\rho}} s_{x x} \\
-\frac{1}{\rho_{3}} q_{x}-\frac{\delta}{\rho_{3}} v_{x} \\
-\frac{\alpha}{\tau} q-\frac{1}{\tau} \theta_{x}
\end{array}\right) .
$$


Now, we consider the following spaces:

$$
\begin{gathered}
H_{*}^{1}(0,1)=\left\{\eta \mid \eta \in H^{1}(0,1): \eta(0)=0\right\}, \quad \tilde{H}_{*}^{1}(0,1)=\left\{\eta \mid \eta \in H^{1}(0,1): \eta(1)=0\right\}, \\
H_{*}^{2}(0,1)=H^{2}(0,1) \cap H_{*}^{1}(0,1), \quad \tilde{H}_{*}^{2}(0,1)=H^{2}(0,1) \cap \tilde{H}_{*}^{1}(0,1)
\end{gathered}
$$

and define the functional space of $\Phi$ as follows:

$$
\mathcal{H}=\tilde{H}_{*}^{1}(0,1) \times L^{2}(0,1) \times H_{*}^{1}(0,1) \times L^{2}(0,1) \times H_{*}^{1}(0,1) \times L^{2}(0,1) \times L^{2}(0,1) \times L^{2}(0,1),
$$

where $\mathcal{H}$ is a Hilbert space endowed with the inner product defined, for $\Phi, \tilde{\Phi} \in \mathcal{H}$, by

$$
\begin{aligned}
(\Phi, \tilde{\Phi})_{\mathcal{H}}= & \rho \int_{0}^{1} u \tilde{u} \mathrm{~d} x+I_{\rho} \int_{0}^{1} v \tilde{v} \mathrm{~d} x+3 I_{\rho} \int_{0}^{1} z \tilde{z} \mathrm{~d} x+\rho_{3} \int_{0}^{1} \theta \tilde{\theta} \mathrm{d} x+\tau \int_{0}^{1} q \tilde{q} \mathrm{~d} x \\
& +G \int_{0}^{1}\left(3 s-\xi-w_{x}\right)\left(3 \tilde{s}-\tilde{\xi}-\tilde{w}_{x}\right) \mathrm{d} x+D \int_{0}^{1} \xi_{x} \tilde{\xi}_{x} \mathrm{~d} x \\
& +4 \gamma \int_{0}^{1} s \tilde{s} \mathrm{~d} x+3 D \int_{0}^{1} s_{x} \tilde{s}_{x} \mathrm{~d} x .
\end{aligned}
$$

Then, the domain of $\mathcal{A}$ is defined by

$$
\begin{array}{r}
D(\mathcal{A})=\left\{\Phi \in \mathcal{H} \mid w \in \tilde{H}_{*}^{2}(0,1), \xi, s \in H_{*}^{2}(0,1), \theta \in \tilde{H}_{*}^{1}(0,1), q \in H_{*}^{1}(0,1),\right. \\
\left.u \in \tilde{H}_{*}^{1}(0,1), v, z \in H_{*}^{1}(0,1), w_{x}(0, t)=\xi_{x}(1, t)=s_{x}(1, t)=0\right\} .
\end{array}
$$

The well-posedness of problem (2.2) is ensured by

Theorem 2.1 [1, Theorem 3.1] Let $\Phi_{0} \in \mathcal{H}$, then problem (2.2) exists a unique weak solution $\Phi \in C\left(\mathbb{R}^{+} ; \mathcal{H}\right)$. Moreover, if $\Phi_{0} \in D(\mathcal{A})$, then

$$
\Phi \in C\left(\mathbb{R}^{+} ; D(\mathcal{A})\right) \cap C^{1}\left(\mathbb{R}^{+} ; \mathcal{H}\right)
$$

Our main result reads as follows:

Theorem 2.2 Let $S(t)=e^{\mathcal{A} t}$ be a $C_{0}$-semigroup of contractions on Hilbert space $\mathcal{H}$. Assume that the stability number $\chi_{\tau} \neq 0$, then the semigroup $S(t)$ on $\mathcal{H}$ is not exponentially stable.

\section{The lack of exponential stability}

In this section, we prove the system is not exponential stable by making use of Prüss theorem to dissipative systems $[6,8]$.

Theorem 3.1 Let $S(t)=e^{\mathcal{A} t}$ be a $C_{0}$-semigroup of contractions on Hilbert space $\mathcal{H}$. Then $S(t)$ is exponentially stable if and only if

$$
\rho(\mathcal{A}) \supset\{i \lambda: \lambda \in \mathbb{R}\} \equiv i \mathbb{R} \quad \text { and } \quad \varlimsup_{|\lambda| \rightarrow \infty}\left\|(i \lambda I-\mathcal{A})^{-1}\right\|_{\mathcal{L}(\mathcal{H})}<\infty
$$

where $\rho(\mathcal{A})$ is the resolvent set of the operator $\mathcal{A}$. 
Proof of Theorem 2.2. We use the above theorem to prove the lack of exponential stability. The main idea of the proof is to show that there exist a sequence of imaginary number $\lambda_{\mu}, \mu \in \mathbb{N}^{+}$ such that

$$
\left\|\left(\lambda_{\mu} I-\mathcal{A}\right)^{-1}\right\|_{\mathcal{L}(\mathcal{H})}<\infty,
$$

which is equivalent to prove that there exist $F_{\mu} \in \mathcal{H}\left(\left\|F_{\mu}\right\|_{\mathcal{H}} \leq 1\right)$ and $V_{\mu} \in D(\mathcal{A})$ with

$$
\left\|\left(\lambda_{\mu} I-\mathcal{A}\right)^{-1} F_{\mu}\right\|_{\mathcal{H}}=\left\|V_{\mu}\right\|_{\mathcal{H}} \rightarrow \infty
$$

Hence, we obtain that

$$
\left(\lambda_{\mu}-\mathcal{A}\right) V_{\mu}=F_{\mu}
$$

Rewriting the spectral equation (3.1) in term of its components, we have $\lambda_{\mu}=\lambda$,

$$
\left\{\begin{array}{l}
\lambda v_{1}-v_{2}=f_{1} \\
\lambda \rho v_{2}-G \partial_{x x} v_{1}-G \partial_{x} v_{3}+3 G \partial_{x} v_{5}=\rho f_{2} \\
\lambda v_{3}-v_{4}=f_{3} \\
\lambda I_{\rho} v_{4}+G \partial_{x} v_{1}+G v_{3}-D \partial_{x x} v_{3}-3 G v_{5}+\delta \partial_{x} v_{7}=I_{\rho} f_{4} \\
\lambda v_{5}-v_{6}=f_{5} \\
3 \lambda I_{\rho} v_{6}-3 G \partial_{x} v_{1}-3 G v_{3}+(9 G+4 \gamma) v_{5}-3 D \partial_{x x} v_{5}+4 \beta v_{6}=3 I_{\rho} f_{6} \\
\lambda \rho_{3} v_{7}+\partial_{x} v_{8}+\delta \partial_{x} v_{4}=\rho_{3} f_{7} \\
\lambda \tau v_{8}+\alpha v_{8}+\partial_{x} v_{7}=\tau f_{8}
\end{array}\right.
$$

where $V=\left(v_{1}, v_{2}, v_{3}, v_{4}, v_{5}, v_{6}, v_{7}, v_{8}\right)^{T} \in D(\mathcal{A})$ and $F=\left(f_{1}, f_{2}, f_{3}, f_{4}, f_{5}, f_{6}, f_{7}, f_{8}\right)^{T} \in \mathcal{H}$.

Next, choosing $f_{1}=f_{3}=f_{4}=f_{5}=f_{6}=f_{7}=f_{8}=0$ and $f_{2}=\frac{1}{\rho} \cos \left(\frac{\mu \pi}{2} x\right)$, we obtain

$$
\left\{\begin{array}{l}
\lambda^{2} \rho v_{1}-G \partial_{x x} v_{1}-G \partial_{x} v_{3}+3 G \partial_{x} v_{5}=\cos \left(\frac{\mu \pi}{2} x\right) \\
\lambda^{2} I_{\rho} v_{3}+G \partial_{x} v_{1}+G v_{3}-D \partial_{x x} v_{3}-3 G v_{5}+\delta \partial_{x} v_{7}=0 \\
\lambda^{2} I_{\rho} v_{5}-G \partial_{x} v_{1}-G v_{3}+\left(3 G+\frac{4 \gamma}{3}\right) v_{5}-D \partial_{x x} v_{5}+\lambda \frac{4 \beta}{3} v_{5}=0 \\
\lambda \rho_{3} v_{7}+\partial_{x} v_{8}+\lambda \delta \partial_{x} v_{3}=0 \\
\lambda \tau v_{8}+\alpha v_{8}+\partial_{x} v_{7}=0
\end{array}\right.
$$

Then, taking the boundary conditions into consideration, we set

$$
\begin{gathered}
v_{1}=A \cos \left(\frac{\mu \pi}{2} x\right), \quad v_{3}=B \sin \left(\frac{\mu \pi}{2} x\right), \quad v_{5}=C \sin \left(\frac{\mu \pi}{2} x\right), \\
v_{7}=E \cos \left(\frac{\mu \pi}{2} x\right), \quad v_{8}=F \sin \left(\frac{\mu \pi}{2} x\right) .
\end{gathered}
$$


Consequently, (3.3) becomes

$$
\left\{\begin{array}{l}
\left(\lambda^{2} \rho+G\left(\frac{\mu \pi}{2}\right)^{2}\right) A-G\left(\frac{\mu \pi}{2}\right) B+3 G\left(\frac{\mu \pi}{2}\right) C=1 \\
-G\left(\frac{\mu \pi}{2}\right) A+\left(\lambda^{2} I_{\rho}+G+D\left(\frac{\mu \pi}{2}\right)^{2}\right) B-3 G C-\delta\left(\frac{\mu \pi}{2}\right) E=0 \\
G\left(\frac{\mu \pi}{2}\right) A-G B+\left(\lambda^{2} I_{\rho}+3 G+\frac{4 \gamma}{3}+\lambda \frac{4 \beta}{3}+D\left(\frac{\mu \pi}{2}\right)^{2}\right) C=0 \\
\lambda \delta\left(\frac{\mu \pi}{2}\right) B+\lambda \rho_{3} E+\left(\frac{\mu \pi}{2}\right) F=0 \\
-\left(\frac{\mu \pi}{2}\right) E+(\lambda \tau+\alpha) F=0 .
\end{array}\right.
$$

Now, we take $\lambda^{2} \rho+G\left(\frac{\mu \pi}{2}\right)^{2}=0$ such that $\lambda=i \sqrt{\frac{G}{\rho}}\left(\frac{\mu \pi}{2}\right)$, then, (3.4) can be rewritten as

$$
\left\{\begin{array}{l}
-G\left(\frac{\mu \pi}{2}\right) B+3 G\left(\frac{\mu \pi}{2}\right) C=1, \\
-G\left(\frac{\mu \pi}{2}\right) A+\left(-\frac{I_{\rho} G}{\rho}\left(\frac{\mu \pi}{2}\right)^{2}+G+D\left(\frac{\mu \pi}{2}\right)^{2}\right) B-3 G C-\delta\left(\frac{\mu \pi}{2}\right) E=0 \\
G\left(\frac{\mu \pi}{2}\right) A-G B+\left(-\frac{I_{\rho} G}{\rho}\left(\frac{\mu \pi}{2}\right)^{2}+3 G+\frac{4 \gamma}{3}+i \frac{4 \beta}{3} \sqrt{\frac{G}{\rho}}\left(\frac{\mu \pi}{2}\right)+D\left(\frac{\mu \pi}{2}\right)^{2}\right) C=0 \\
i \delta \sqrt{\frac{G}{\rho}}\left(\frac{\mu \pi}{2}\right)^{2} B+i \rho_{3} \sqrt{\frac{G}{\rho}}\left(\frac{\mu \pi}{2}\right) E+\left(\frac{\mu \pi}{2}\right) F=0 \\
-\left(\frac{\mu \pi}{2}\right) E+\left(i \tau \sqrt{\frac{G}{\rho}}\left(\frac{\mu \pi}{2}\right)+\alpha\right) F=0 .
\end{array}\right.
$$

From $(3.5)_{5}$, we have

$$
F=\frac{\left(\frac{\mu \pi}{2}\right)}{i \tau \sqrt{\frac{G}{\rho}}\left(\frac{\mu \pi}{2}\right)+\alpha} E .
$$

Substituting (3.6) into $(3.5)_{4}$ yields

$$
E=\frac{\frac{G \delta \tau}{\rho}\left(\frac{\mu \pi}{2}\right)^{3}-i \delta \alpha \sqrt{\frac{G}{\rho}}\left(\frac{\mu \pi}{2}\right)^{2}}{\left(1-\frac{G \tau \rho_{3}}{\rho}\right)\left(\frac{\mu \pi}{2}\right)^{2}+i \rho_{3} \alpha \sqrt{\frac{G}{\rho}}\left(\frac{\mu \pi}{2}\right)} B .
$$

Combining $(3.5)_{2}$ and $(3.5)_{3}$, we obtain

$$
\begin{aligned}
& \left(-\frac{I_{\rho} G}{\rho}\left(\frac{\mu \pi}{2}\right)^{2}+D\left(\frac{\mu \pi}{2}\right)^{2}\right) B \\
& +\left(-\frac{I_{\rho} G}{\rho}\left(\frac{\mu \pi}{2}\right)^{2}+\frac{4 \gamma}{3}+i \frac{4 \beta}{3} \sqrt{\frac{G}{\rho}}\left(\frac{\mu \pi}{2}\right)+D\left(\frac{\mu \pi}{2}\right)^{2}\right) C-\delta\left(\frac{\mu \pi}{2}\right) E=0 .
\end{aligned}
$$

Then, replacing (3.7) in (3.8), we get

$$
C=-\frac{\left(-\frac{I_{\rho} G}{\rho}\left(\frac{\mu \pi}{2}\right)^{2}+D\left(\frac{\mu \pi}{2}\right)^{2}\right)+\frac{\frac{-G \delta^{2} \tau}{\rho}\left(\frac{\mu \pi}{2}\right)^{4}+i \delta^{2} \alpha \sqrt{\frac{G}{\rho}}\left(\frac{\mu \pi}{2}\right)^{3}}{\left(1-\frac{G \tau \rho_{3}}{\rho}\right)\left(\frac{\mu \pi}{2}\right)^{2}+i \rho_{3} \alpha \sqrt{\frac{G}{\rho}\left(\frac{\mu \pi}{2}\right)}}}{-\frac{I_{\rho} G}{\rho}\left(\frac{\mu \pi}{2}\right)^{2}+\frac{4 \gamma}{3}+i \frac{4 \beta}{3} \sqrt{\frac{G}{\rho}}\left(\frac{\mu \pi}{2}\right)+D\left(\frac{\mu \pi}{2}\right)^{2}} B=-\frac{A_{\mu}}{C_{\mu}} B .
$$


Gathering (3.5) ${ }_{3}$ and (3.9), we obtain

$$
A=\frac{G+A_{\mu}+3 G \frac{A_{\mu}}{C_{\mu}}}{G\left(\frac{\mu \pi}{2}\right)} B
$$

Next, substituting (3.9) into $(3.5)_{1}$, we arrive at

$$
B=-\frac{1}{G\left(\frac{\mu \pi}{2}\right)+\frac{3 G A_{\mu}}{C_{\mu}}\left(\frac{\mu \pi}{2}\right)} .
$$

Let $\chi=\frac{D}{G}-\frac{I_{\rho}}{\rho}, \chi_{1}=1-\frac{\tau \rho_{3} G}{\rho}$. In case $\chi_{1}=0$, we get

$$
B \rightarrow \frac{i \rho_{3} \alpha \chi}{3 \tau \delta^{2} \sqrt{\frac{G}{\rho}}\left(\frac{\mu \pi}{2}\right)^{2}}, \text { for } \mu \text { large. }
$$

Then

$$
A \rightarrow-\frac{\chi}{3 G}, \quad B \rightarrow 0, \quad C \rightarrow 0, \quad E \rightarrow \frac{\chi}{3 \delta}, \quad F \rightarrow \frac{\chi}{3 i \delta \tau \sqrt{\frac{G}{\rho}}}, \quad \mu \rightarrow \infty .
$$

Thus, we obtain

$$
\begin{aligned}
\left\|V_{\mu}\right\|_{\mathcal{H}}^{2} & \geq G \int_{0}^{1}\left(3 v_{5}-v_{3}-v_{1 x}\right)^{2} \mathrm{~d} x \\
& =G \int_{0}^{1}\left(3 C \sin \left(\frac{\mu \pi}{2} x\right)-B \sin \left(\frac{\mu \pi}{2} x\right)+A\left(\frac{\mu \pi}{2}\right) \sin \left(\frac{\mu \pi}{2} x\right)\right)^{2} \mathrm{~d} x \\
& =G\left(3 C-B+A\left(\frac{\mu \pi}{2}\right)\right)^{2} \int_{0}^{1}\left(\sin \left(\frac{\mu \pi}{2} x\right)\right)^{2} \mathrm{~d} x \\
& \approx \frac{\chi^{2}}{18 G}\left(\frac{\mu \pi}{2}\right)^{2} \rightarrow \infty, \text { as } \mu \rightarrow \infty
\end{aligned}
$$

In case $\chi_{1} \neq 0$, we have

$$
B \rightarrow-\frac{\chi \chi_{1}}{\left(3 I_{\rho} \chi_{\tau}+\chi \chi_{1} G\right)\left(\frac{\mu \pi}{2}\right)}, \quad \text { for } \mu \text { large. }
$$

Therefore

$$
\begin{gathered}
A \rightarrow-\frac{I_{\rho} \chi \chi_{\tau}}{3 G I_{\rho} \chi_{\tau}+\chi \chi_{1} G^{2}}, B \rightarrow 0, C \rightarrow 0, E \rightarrow-\frac{\tau \delta G \chi}{3 \rho I_{\rho} \chi_{\tau}+\rho G \chi_{\chi_{1}}}, \\
F \rightarrow \frac{i G \delta \chi}{\left(3 \rho I_{\rho} \chi_{\tau}+\rho \chi \chi_{1} G\right) \sqrt{\frac{G}{\rho}}}, \mu \rightarrow \infty .
\end{gathered}
$$

Consequently,

$$
\begin{aligned}
\left\|V_{\mu}\right\|_{\mathcal{H}}^{2} & \geq G \int_{0}^{1}\left(3 v_{5}-v_{3}-v_{1 x}\right)^{2} \mathrm{~d} x \\
& =G \int_{0}^{1}\left(3 C \sin \left(\frac{\mu \pi}{2} x\right)-B \sin \left(\frac{\mu \pi}{2} x\right)+A\left(\frac{\mu \pi}{2}\right) \sin \left(\frac{\mu \pi}{2} x\right)\right)^{2} \mathrm{~d} x \\
& =G\left(3 C-B+A\left(\frac{\mu \pi}{2}\right)\right)^{2} \int_{0}^{1}\left(\sin \left(\frac{\mu \pi}{2} x\right)\right)^{2} \mathrm{~d} x
\end{aligned}
$$




$$
\approx \frac{G I_{\rho}^{2} \chi^{2} \chi_{\tau}^{2}}{2\left(3 G I_{\rho} \chi_{\tau}+\chi \chi_{1} G^{2}\right)^{2}}\left(\frac{\mu \pi}{2}\right)^{2} \rightarrow \infty, \text { as } \mu \rightarrow \infty .
$$

From the above, it is easy to see that

$$
\left\|V_{\mu}\right\|_{\mathcal{H}} \rightarrow \infty, \text { as } \mu \rightarrow \infty
$$

which is the desired conclusion.

\section{Acknowledgments}

This work was supported by the National Natural Science Foundation of China (Grant No. 11771216, 11301277), the Natural Science Foundation of Jiangsu Province (Grant No. BK20151523), the Six Talent Peaks Project in Jiangsu Province (Grant No. 2015-XCL-020), and the Qing Lan Project of Jiangsu Province.

\section{References}

[1] T. A. Apalara, Uniform stability of a laminated beam with structural damping and second sound, Z. Angew. Math. Phys. 68 (2017), no. 2, 68:41.

[2] T. A. Apalara, S. A. Messaoudi and A. A. Keddi, On the decay rates of Timoshenko system with second sound, Math. Methods Appl. Sci. 39 (2016), no. 10, 2671-2684.

[3] F. Dell'Oro, Asymptotic stability of thermoelastic systems of Bresse type, J. Differential Equations 258 (2015), no. 11, 3902-3927.

[4] H. D. FernÁndez Sare and R. Racke, On the stability of damped Timoshenko systems: Cattaneo versus Fourier law, Arch. Ration. Mech. Anal. 194 (2009), no. 1, 221-251.

[5] A. A. Keddi, T. A. Apalara and S. A. Messaoudi, Exponential and Polynomial Decay in a Thermoelastic-Bresse System with Second Sound, Appl Math Optim (2016), in press, doi:10.1007/s00245-016-9376-y.

[6] J. Prüss, On the spectrum of $C_{0}$-semigroups, Trans. Amer. Math. Soc. 284 (1984), no. 2, 847-853.

[7] M. L. Santos, D. S. Almeida Júnior and J. E. Muñoz Rivera, The stability number of the Timoshenko system with second sound, J. Differential Equations 253 (2012), no. 9, 27152733.

[8] M. L. Santos, D. S. Almeida Júnior and J. E. Muñoz Rivera, Bresse system with Fourier law on shear force, Adv. Differential Equations 21 (2016), no. 1-2, 55-84. 